\title{
Nobel Lecture: Spontaneous symmetry breaking in particle physics: A case of cross fertilization*
}

\author{
Yoichiro Nambu \\ University of Chicago, The Enrico Fermi Institute, Chicago, Illinois 60637, USA \\ (Published 15 July 2009; corrected 24 November 2010) \\ DOI: $10.1103 /$ RevModPhys.81.1015
}

I will begin by a short story about my background. I studied physics at the University of Tokyo. I was attracted to particle physics because of the three famous names, Nishina, Tomonaga, and Yukawa, who were the founders of particle physics in Japan. But these people were at different institutions than mine. On the other hand, condensed matter physics was pretty good at Tokyo. I got into particle physics only when I came back to Tokyo after the war. In hindsight, though, I must say that my early exposure to condensed matter physics has been quite beneficial to me.

Particle physics is an outgrowth of nuclear physics which began in the early 1930s with the discovery of the neutron by Chadwick, the invention of the cyclotron by Lawrence, and the "invention" of meson theory by Yukawa (Nambu, 2007). The appearance of an everincreasing array of new particles in the subsequent decades, and the advances in quantum field theory gradually led to our understanding of the basic laws of nature, culminating in the present standard model.

When we faced those new particles, our first attempts were to make sense out of them by finding some regularities in their properties. They invoked the symmetry principle to classify them. Symmetry in physics leads to a conservation law. Some conservation laws are exact, like energy and electric charge, but these attempts were based on approximate similarities of masses and interactions.

Nevertheless, seeing similarities is a natural and very useful trait of the human mind. The near equality of proton and neutron masses and their interactions led to the concept of isospin $\mathrm{SU}(2)$ symmetry (Heisenberg, 1932). On the other hand, one could also go in the opposite direction, and elevate symmetry to a more elaborate gauged symmetry. Then symmetry will determine the dynamics as well, a most attractive possibility. Thus the beautiful properties of electromagnetism was extended to the SU(2) non-Abelian gauge field (Yang and Mills, 1954). But strong interactions are short range. Giving a mass to a gauge field destroys gauge invariance.

Spontaneous symmetry breaking (SSB), which is the main subject of my talk, is a phenomenon where a sym-

\footnotetext{
*The 2008 Nobel Prize for Physics was shared by Yoichiro Nambu, Makoto Kobayashi, and Toshihide Maskawa. This paper is the text of the address given in conjunction with the award.
}

metry in the basic laws of physics appears to be broken. In fact, it is a very familiar one in our daily life, although the name SSB is not (the name is due to Baker and Glashow, 1962). For example, consider a elastic straight rod standing vertically. It has a rotational symmetry; it looks the same from any horizontal direction. But if one applies increasing pressure to squeeze it, it will bend in some direction, and the symmetry is lost. The bending can occur in principle in any direction since all directions are equivalent. But you do not see it unless you repeat the experiment many times. This is SSB.

The SSB in quantum mechanics occurs typically in a uniform medium consisting of a large number of elements. It is a dynamical effect. Symmetry allows some freedom of action to each of them but the interaction among them forces them, figuratively speaking, to line up like a crowd of people looking in the same direction. Then it is not easy to change the direction wholesale even if it is allowed by the symmetry and hence does not take energy, because the action is not local operator. So the symmetry appears to be lost. It is still possible to recover the lost symmetry by a global operation, but it would amount to a kind of phase transition. Some of the examples are

Physical system Broken symmetry

Ferromagnets

Rotational invariance (with respect to spin)

Crystals Translational and rotational invariance (modulo discrete values)

Superconductors Local gauge invariance (particle number)

SSB in a medium then has the following characteristic properties:

(1) The ground state has a huge degeneracy. A symmetry operation takes one ground state to another.

(2) Only one of the ground states and a whole spectrum of excited states built on it are realized in a given situation.

(3) SSB is, in general, lost at sufficiently high temperatures.

In relativistic quantum field theory, this phenomenon becomes also possible for the entire space-time, for the "vacuum" is not void, but has many intrinsic degrees of 
freedom. In this context, it may play an important role in cosmology. As the universe expands and cools down, it may undergo one or more SSB phase transitions from states of higher symmetries to lower ones, which change the governing laws of physics.

I will now recall the chain of events which led me to the idea of SSB and its application to particle physics. One day in 1956 R. Schrieffer gave us a seminar on what would come to be called the BCS theory (Bardeen et al., 1957) of superconductivity. I was impressed by the boldness of their ansatz for the state vector, but at the same time I became worried about the fact that it did not appear to respect gauge invariance. Soon thereafter Bogoliubov (1958) [this is a fermionic version of transformation which he first introduced in a description of superfluidity (1947)] and Valatin (1958) independently introduced the concept of quasiparticles as fermionic excitations in the BCS medium. The quasiparticles did not carry a definite charge as they were a superposition of electron and hole, with their proportion depending on the momentum. How can one then trust the BCS theory for discussing the electromagnetic properties like the Meissner effect? It actually took two years for me to resolve the problem to my satisfaction. There were a number of people who also addressed the same problem, but I wanted to understand it in my own way. Essentially it is the presence of a massless collective mode, now known by the generic name of Nambu-Goldstone (NG) boson, that saves charge conservation or gauge invariance.

The Bogoliubov-Valatin (BV) quasiparticles are described by the equations (Nambu, 1960a),

$$
\begin{aligned}
& E \psi_{\mathbf{p},+}=\varepsilon_{\mathbf{p}} \psi_{\mathbf{p},+}+\Delta \psi_{-\mathbf{p},-}^{\dagger}, \\
& E \psi_{-\mathbf{p},-}^{\dagger}=-\varepsilon_{\mathbf{p}} \psi_{-\mathbf{p},-}^{\dagger}+\Delta \psi_{\mathbf{p},+}, \\
& E=\sqrt{\varepsilon_{\mathbf{p}}^{2}+\Delta^{2}} .
\end{aligned}
$$

Here $\psi_{\mathbf{p},+}$ and $\psi_{-\mathbf{p},-}^{\dagger}$ are the wave functions for an electron and a hole of momentum $p$ and spin + or,$- \varepsilon_{p}$ is the kinetic energy relative to the Fermi energy, and $2 \Delta$ is the energy gap. In terms of spinlike matrices $\tau_{i}$, the corresponding Hamiltonian and the charge current are

$$
\begin{aligned}
& H_{0}=\varepsilon_{\mathbf{p}} \Psi^{\dagger} \tau_{3} \Psi+\Delta \Psi^{\dagger} \tau_{1} \Psi, \\
& \rho_{0}=\Psi^{\dagger} \tau_{3} \Psi, \quad \mathbf{j}_{0}=\Psi^{\dagger}(\mathbf{p} / m) \Psi .
\end{aligned}
$$

The BV ground state is $\Psi_{\mathbf{p}}|0\rangle=0$ for all $\mathbf{p}$. The charge does not commute with $H_{0}$, and the continuity equation does not hold, which is the problem. But it has turned out that the same interaction that led to the BCS-BV ground state also leads to collective excitations $f$, which contributes to the charge current and restores the continuity equation. The correct expression is

$$
\begin{aligned}
& \rho \simeq \rho_{0}+\frac{1}{\alpha^{2}} \partial_{t} f, \\
& \mathbf{j} \simeq \mathbf{j}_{0}-\nabla f
\end{aligned}
$$

$$
\left(\nabla^{2}-\frac{1}{\alpha^{2}} \partial_{t}^{2}\right) f \simeq-2 \Delta \Psi^{\dagger} \tau_{2} \Psi
$$

$f$ represents the NG mode. Physically, it corresponds to excitations that tend to restore the lost symmetry. Its energy goes to zero in the long wavelength limit as it corresponds to the global symmetry operation. It also happens that the above NG mode also mixes with the Coulomb interaction among the electrons because of their common long-range nature, and turns into the well-known plasmons with

$$
\omega^{2}=e^{2} n / m,
$$

where $e, n$, and $m$ are, respectively, the charge, density, and mass of the electron.

The formal similarity of the BV equation to the Dirac equation naturally led me to transport the BCS theory to particle physics (Nambu, 1960b). The gap $\Delta$ goes over to the mass $M$, which breaks chirality $\sim \gamma_{5}$ rather than the ordinary charge $\sim 1$. The axial current is the analog of the electromagnetic vector current in the BCS theory. If chirality is a broken symmetry, the matrix elements of the axial current between nucleon states of fourmomentum $p$ and $p^{\prime}$ should have the form

$$
\Gamma_{\mu 5}\left(p^{\prime}, p\right)=\left(\gamma_{\mu} \gamma_{5}-2 M \gamma_{5} q_{\mu} / q^{2}\right) F\left(q^{2}\right), \quad q_{\mu}=p_{\mu}^{\prime}-p_{\mu} .
$$

So chiral symmetry is compatible with a finite nucleon mass $M$ provided that there exists a massless pseudoscalar NG boson. In reality, there is a pseudoscalar pion, and the vector and axial vector interactions that appear in weak decays of the nucleons and the pion had the properties

$$
g_{V} \simeq g_{A}, \quad g_{\pi} \simeq \sqrt{2} M g_{A} / G,
$$

where $g_{V}$ and $g_{A}$ are vector and axial vector couplings of the nucleon, $g_{\pi}$ is axial coupling for the pion, $G$ is the pion-nucleon coupling, and $M$ is the nucleon mass. The second equation was known as the Goldberger-Treiman relation (Goldberger and Treiman, 1958), and it implies that the matrix element of the axial vector part of nucleon decay is

$$
\Gamma_{\mu A} \simeq\left(\gamma_{\mu} \gamma_{5}-2 M \gamma_{5} q_{\mu}\right) /\left(q^{2}-m_{\pi}^{2}\right),
$$

which differs from Eq. (5) by the presence of pion mass. In view of the smallness of $m_{\pi}$ compared to $M$, I made the hypothesis that the axial current is an approximately conserved quantity, the nucleon mass is generated by an SSB of chirality, and the pion is the corresponding NG boson which should become massless in the limit of exact conservation. (Proton and neutron masses should also become equal.)

The first presentation of the idea was made in Nambu (1960a, 1960b); the model system I worked out with Jona-Lasinio (Nambu and Jona-Lasinio (1961a, 1961b) is a concrete realization of the proposed SSB. It has the form similar to the BCS model 


$$
L=-\bar{\psi} \gamma^{\mu} \partial_{\mu} \psi+g\left[(\bar{\psi} \psi)^{2}-\left(\bar{\psi} \gamma_{5} \psi\right)^{2}\right],
$$

which is invariant against the particle number and chiral transformations,

$$
\begin{aligned}
& \psi \rightarrow \exp (i \alpha) \psi, \quad \bar{\psi} \rightarrow \bar{\psi} \exp (-i \alpha), \\
& \psi \rightarrow \exp \left(i \gamma_{5} \alpha\right) \psi, \quad \bar{\psi} \rightarrow \bar{\psi} \exp \left(i \gamma_{5} \alpha\right) .
\end{aligned}
$$

After SSB, the "nucleon" acquires a mass $M$ $\sim 2 g\langle\bar{\psi} \psi\rangle$. Although the model is nonrenormalizable, it is easy to demonstrate the SSB mechanism. The generated mass $M$ is determined by the "gap equation"

$$
\frac{2 \pi^{2}}{g \Lambda^{2}}=1-\frac{M^{2}}{\Lambda^{2}} \ln \left(1+\frac{\Lambda^{2}}{M^{2}}\right),
$$

where $\Lambda$ is a cutoff. Bound states of nucleon-antinucleon (meson) and nucleon-nucleon (dibaryon) pairs of spin 0 and 1 were also found. In particular, the masses of $0^{-}$ $\left(\sim \bar{\psi} \gamma_{5} \psi\right)$ and $0^{+}(\sim \bar{\psi} \psi)$ mesons are found to be 0 and $2 M$, respectively. A more realistic two-flavor model was also considered by generalizing Eq. (8) to

$$
L=-\bar{\psi} \gamma^{\mu} \partial_{\mu} \psi+g\left[(\bar{\psi} \psi)^{2}-\Sigma_{i}\left(\bar{\psi} \gamma_{5} \tau_{i} \psi\right)\left(\bar{\psi} \gamma_{5} \tau_{i} \psi\right)\right],
$$

with a similar gap equation. We get an isovector $0^{-}$pion and a isoscalar $0^{+}$. The actual pion mass was generated by a small explicit bare mass in the Lagrangian of the order of $5 \mathrm{MeV}$. This also induced a change in axial coupling constant $g_{A}$ in the right direction.

Other examples of the BCS-type SSB are ${ }^{3} \mathrm{He}$ superfluidity and nucleon pairing in nuclei (Arima and Iachello, 1975, 1976). In general, there exist simple mass relations among the fermion and the boson in BCS-type theories (Nambu, 1985).

The BCS theory also accounts for the generation of the London mass for the electromagnetic field. This problem is made simple in terms of the Higgs scalar field (Anderson, 1963; Englert and Brout, 1964; Higgs, 1964). The relativistic analog of the London relation is, in momentum space,

$$
\begin{aligned}
& j_{\mu}(q)=K_{\mu \nu}(q) A^{\nu}, \\
& K_{\mu \nu}=\left(\delta_{\mu \nu}-q_{\mu} q_{\nu} / q^{2}\right) K\left(q^{2}\right), \\
& K\left(q^{2}\right) \simeq q^{2} /\left(q^{2}-m^{2}\right) .
\end{aligned}
$$

The third relation shows the massless NG boson turning into a massive "plasmon," a process corresponding to Eq. (4). This was successfully applied to weak gauge field in the Weinberg-Salam (WS) theory (Weinberg, 1967; Salam, 1968) of electroweak unification. The fermion masses are also generated and break chiral invariance. These so-called current masses for the up and down quarks play the role of the bare mass in the Nambu-Jona-Lasinio model.

In the current standard model of particle physics, the NJL model may be regarded as an effective theory for the QCD with respect to generation of the so-called constituent masses. One is interested in the low energy de- grees of freedom on a scale smaller than some cutoff $\Lambda \sim 1 \mathrm{GeV}$. The short distance dynamics above $\Lambda$ as well as the confinement may be treated as a perturbation The problem has been extensively studied by many people. The Lagrangian adopted by Hatsuda and Kunihiro (1994) is

$$
L_{\mathrm{QCD}} \approx L_{\mathrm{NJL}}+L_{\mathrm{KMT}}+\delta L,
$$

$L_{\mathrm{NJL}}$ is for the quarks, and contains "current mass" terms. $L_{\mathrm{KMT}}$ refers to the Kabayashi-Maskawa-'t Hooft chiral anomaly

$$
L_{\mathrm{KMT}}=g_{D}\left\{\operatorname{det}\left[\bar{q}_{i}\left(1-\gamma_{5}\right) q_{j}\right]+\text { H.c. }\right\} .
$$

Both of them contribute to the explicit breaking of chiral invariance. ( $\delta L$ contains the effects of confinement and one gluon exchange.) The WS theory resembles the Ginzburg-Landau (1950) description of superconductivity which was shown to follow from the BCS theory by Gor'kov (1959). In the same way the NJL model goes over to the model of Gell-Mann and Lévy (1960). If this analogy turns out real, the Higgs field might be an effective description of underlying dynamics.

Finally, I will end this lecture with a comment on the mass hierarchy problem. Hierarchical structure is an outstanding feature of the universe. The masses of known fundamental fermions also make a hierarchy stretching 11 orders of magnitude. Mass is not quantized in a simple regular manner like charge and spin. Mass is a dynamical quantity since it receives contributions from interactions. But we do not see yet a pattern like those in the hydrogen atom which led to quantum mechanics, or the Regge trajectories which led to the dual string picture.

The BCS mechanism seems relevant to the problem, as was remarked earlier. It generates a mass gap for fermions, plus the Nambu-Goldstone and Higgs modes as low-lying bosons. The bosons may act in turn as an agent for further SSB, leading to the possibility of hierarchical SSB or "tumbling" (Raby et al., 1980). In fact we already have examples of it (Nambu, 1998):

(1) The chain atoms-crystal-phononsuperconductivity. The NG mode for crystal formation is the phonon which induces the Cooper pairing of electrons to cause superconductivity.

(2) The chain QCD-chiral SSB of quarks and baryons $-\pi, \sigma$, and other mesons-nuclei formation and nucleon pairing-nuclear collective modes. No further elaboration would be required.

I am greatly thankful to G. Jona-Lasinio for his help in the planning of the lecture.

\section{REFERENCES}

Anderson, P. W., 1963, Phys. Rev. 130, 439.

Arima, A., and F. Iachello, 1975, Phys. Rev. Lett. 35, 1069.

Arima, A., and F. Iachello, 1976, Ann. Phys. 99, 253. 
Baker, M., and S. L. Glashow, 1962, Phys. Rev. 128, 2462.

Bardeen, J., L. N. Cooper, and J. R. Schrieffer, 1957, Phys. Rev. 108, 1175.

Bogoliubov, N. N., 1947, J. Phys. 11, 23.

Bogoliubov, N. N., 1958, J. Exp. Theor. Phys. 34, 58.

Englert, F., and R. Brout, 1964, Phys. Rev. Lett. 13, 321.

Gell-Mann, M., and M. L. Lévy, 1960, Nuovo Cimento 16, 705.

Ginzburg, V. I., and L. D. Landau, 1950, J. Exp. Theor. Phys. 20, 1064

Goldberger, M. L., and S. Treiman, 1958, Phys. Rev. 110, 1178. Gor'kov, L. P., 1959, J. Exp. Theor. Phys. 36, 1918.

Hatsuda, T., and T. Kunihiro, 1994, Phys. Rep. 247, 221.

Higgs, P. W., 1964, Phys. Rev. Lett. 13, 508.

Heisenberg, W., 1932, Z. Phys. 771.

Nambu, Y., 1960a, Phys. Rev. 117, 648.
Nambu, Y., 1960b, Phys. Rev. Lett. 4, 380.

Nambu, Y., 1960c, Proceedings of the Midwest Conference on

Theoretical Physics (Purdue University, Lafayette, IN), p. 1.

Nambu, Y., 1985, Physica D 15, 147.

Nambu, Y., 1998, Nucl. Phys. A 638, 35c.

Nambu, Y., 2007, J. Phys. Soc. Jpn. 76, 111002.

Nambu, Y., and G. Jona-Lasinio, 1961a, Phys. Rev. 122, 345.

Nambu, Y., and G. Jona-Lasinio, 1961b, Phys. Rev. 124, 246.

Raby, S., S. Dimopoulos, and L. Susskind, 1980, Nucl. Phys. B 169, 373.

Salam, A., 1968, Elementary Particles (Almqvyst, Forlag AB) p. 167.

Valatin, G., 1958, Nuovo Cimento 7, 843.

Weinberg, S., 1967, Phys. Rev. Lett. 19, 1264.

Yang, C. N., and R. L. Mills, 1954, Phys. Rev. 96, 191. 